\title{
A Multi-hop ARQ Protocol for Underwater Acoustic Networks
}

\author{
Hwee-Pink Tan ${ }^{*}$, Winston K. G. Seah ${ }^{\dagger}$ and Linda Doyle ${ }^{*}$ \\ ${ }^{*}$ Center for Telecommunications Value-chain Research \\ Trinity College Dublin (Republic of Ireland) \\ $\dagger$ Network Technology Department, Institute for Infocomm Research (Singapore) \\ Email: \{tanhp@tcd.ie, winston@i2r.a-star.edu.sg and ledoyle@tcd.ie\}
}

\begin{abstract}
Underwater acoustic networks are envisaged to be the enabling technology for oceanographic data collection, pollution monitoring, offshore exploration and tactical surveillance applications. Unique characteristics of underwater acoustic channels such as large propagation delays and high bit error rates pose a challenge to designing reliable and efficient communication protocols. In this paper, we propose an opportunistic acknowledgement scheme suited for Stop and Wait ARQ protocols and demonstrate using simulations that it achieves better latency and energy efficiency than traditional non-opportunistic schemes for both one and two-dimensional multi-hop acoustic channels.
\end{abstract}

\section{INTRODUCTION}

Many applications have been envisaged for underwater sensor networks, including seismic monitoring, tactical surveillance and equipment monitoring and fault detection. To realize these applications, we may borrow many design principles and tools from ongoing, ground-based terrestrial sensor research. Acoustic communication is a promising candidate for underwater communication since radio is not suitable for underwater usage because of extremely limited propagation. However, there exist some fundamental differences between the radio and acoustic media, e.g., (a) long and variable propagation delay and (b) high and variable bit error rate (BER) in the latter that pose research challenges in underwater sensor networking [1], [2].

In a typical underwater sensor network, sensor nodes are interconnected via wireless links to one or more underwater sinks that are responsible for reliably relaying data to a surface station. While direct communication between sensor and sink nodes (long range, high power) is simple, multi-hop (short-range, low power) communication using complex protocols may be necessary, for example, in military surveillance applications, where operational covertness and low power consumption are critical requirements.

To establish reliable multi-hop communication, an automatic repeat request ( $A R Q$ ) procedure is required to manage the retransmission of erroneously received / lost data packets. Since current acoustic modem technology typically supports half-duplex mode, the method of choice for current ARQ implementations is the simple Stop and Wait (S\&W) protocol where each transmitted packet has to be acknowledged before the next packet can be transmitted. This can be simply achieved by explicitly transmitting an acknowledgement packet (typically of much smaller size than data packet) per successfully received data packet in a single-hop link.

However, in a multi-hop channel, due to the broadcast nature of the medium, if a node transmits a packet and hears its next-hop neighbour transmitting it forward, it is an implicit acknowledgement that the packet has been successfully received by its neighbour, hence nullifying the need for explicit acknowledgement. While this may be more efficient (e.g. in terms of bits transmitted per data packet and/or waiting time for acknowledgements) than explicit acknowledgement in terrestrial wireless links with very low BER, it may not be so for acoustic links with substantially higher BER. In addition, while acknowledgement may be implemented on a per-hop or end-to-end basis, the former is expected to be more efficient for high BER acoustic links.

Hence, in this paper, we propose a per-hop hybrid implicit/explicit acknowledgement scheme for S\&W ARQ in a multi-hop acoustic channel, and demonstrate its efficacy in one- and two-dimensional architectures suitable for long range, underwater surveillance applications.

\section{RELATED WORK}

Variants of S\&W have been proposed [3], [4], [5], [6] for single-hop communication links to improve its efficiency by transmitting blocks of packets, rather than a single packet, thus making better utilisation of the time spent in waiting for the acknowledgements. However, to the best of our knowledge, ARQ mechanisms for multi-hop communication is much less studied [7], [8].

Recently, in [9], the author conducted a statistical analysis of protocol efficiency for a class of S\&W protocols, leading to an optimal packet size in terms of hop-length, bit rate and expected BER under typical underwater channels. As in [3], [4], [5], [6], this work considers S\&W schemes based on transmitting groups of packets for which selective acknowledgements are generated. A similar analysis was conducted in [10], which additionally accounted for cross-layer interactions with medium access control layers and forward error correction. However, the above analyses were conducted over single-hop acoustic links.

On the other hand, multi-hop end-to-end ARQ (e2e-ARQ) concepts (as opposed to $\mathrm{S} \& \mathrm{~W}$ which is a per-hop (PH-ARQ) mechanism) were proposed recently in [7], [8] primarily to 
handle node mobility in beyond $3 \mathrm{G}$ terrestrial access networks. An e2e RelayARQ protocol is proposed in [7] that allows relay or intermediate nodes to seamlessly "leave and join" without breaking the end-to-end connection by maintaining a common protocol state between all nodes. A multi-hop ARQ mechanism $(M-A R Q)$ is proposed in [8] by coupling a $\mathrm{PH}-$ ARQ protocol with an e2e-ARQ. Although e2e-ARQ protocols may perform better in terms of reliability and efficiency than PH-ARQ, the added complexity may not be suited for implementation in underwater networks comprising energystarved sensor nodes. In addition, node mobility may not be a critical issue in underwater networks. Therefore, we focus our efforts on devising a S\&W protocol suited for multi-hop communication in underwater networks.

\section{STOP AND WAIT ARQ FOR MULTI-HOP ACOUSTIC COMMUNICATION}

\section{A. System Model and Problem Definition}

Consider an $n$-hop acoustic channel comprising a source node, sink node and $n-1$ relay nodes as shown in Fig. 1 (a). One of the distinguishing features of underwater acoustic communication is spatially-varying channels, which is characterized here by the BER along each hop $j, p_{j}$.

When a packet of $b$ bits is transmitted over hop $j$, the corresponding packet error probability $p_{j}(b)$, is:

$$
p_{j}(b)=1-\left(1-p_{j}\right)^{b}
$$

The time required for this packet to arrive at its intended recipient node (either node $j-1$ or $j+1$ ) comprises the transmission delay $t=b / r$ and the propagation delay, $t_{P}=/ / c$, where $r$ is the transmission rate of the channel, $l$ is the hop-length (m) and $c$ is the speed of sound $(\mathrm{m} / \mathrm{s})$.

We consider two types of packets in this study, namely data packets of $b_{D}$ bits and acknowledgement (ACK) packets of $b_{A}\left(<<b_{D}\right)$ bits each, with transmission times given by $t_{D}=$ $b_{D} / r$ and $t_{A}=b_{A} / r$ respectively.

\section{B. Stop and Wait (S\&W) $A R Q$}

With S\&W ARQ, the transmitter sends a packet and waits for the acknowledgment. Once it arrives, the transmitter moves on to a new packet; otherwise, if it does not arrive within a pre-specified duration, called the time-out, the packet is retransmitted. Due to the high BER of acoustic channels, the acknowledgement may be lost and this will trigger an unnecessary retransmission after the time-out. To reduce unnecessary transmission (hence, energy consumption) of data packets which have already been received, we apply the following rule:

Rule 1: Whenever a relay node $j$ receives a packet from node $j-1$, it transmits an ACK packet if its previous data transmission is already acknowledged. On the other hand, (non-relay) node $1(n+1)$ only transmits data (ACK) packets.

The design of the acknowledgement procedure is important since, in addition to energy-efficiency, it also determines the latency-efficiency of the ARQ mechanism, where a longer waiting time before the next packet can be transmitted indicates lower efficiency. The acknowledgement procedure can be done implicitly through the data packet itself or explicitly through an ACK packet. These mechanisms will be described in the subsequent sections.

1) Implicit Acknowledgement (IMP): Consider the transmission of a data packet ( $b_{D}$ bits) over hop $j$ from node $j$ to node $j+1$. Since the transmission is isotropic, this packet may also be received at node $j-1$ and serve as an implicit ACK for its transmission. The corresponding packet error probability over hop $j$-1 (or probability of unsuccessful acknowledgment for node $j-1$ 's transmission) is:

$$
p_{j-1}\left(b_{D}\right)=1-\left(1-p_{j-1}\right)^{b_{D}} .
$$

If node $j-1$ is not acknowledged after a time-out interval, $\tau_{O, \mathrm{IMP}}=2\left(t_{P}+t_{D}\right)$, it retransmits the data packet. We denote this ARQ scheme by IMP.

2) Explicit Acknowledgement (EXP): Since acoustic channels are characterized by high BER, a substantial proportion of implicit ACK packets may be erroneously transmitted, triggering retransmission of data packets that consumes energy and hence should be minimized. One way to achieve this is to define an explicit ACK protocol (EXP) that piggybacks the ACK packet to each data packet. In this way, the corresponding probability of unsuccessful acknowledgment for node $j-1$ 's transmission is reduced to:

$$
p_{j-1}\left(b_{A}\right)=1-\left(1-p_{j-1}\right)^{b_{A}} .
$$

However, the corresponding time-out interval is increased to $\tau_{O, \mathrm{EXP}}=2\left(t_{P}+t_{D}+t_{A}\right)$. Although the improvement in the likelihood of successful acknowledgment comes at the expense of longer time-out intervals, the relative performance of IMP and EXP in terms of latency- and energy-efficiency depends on the choice of system parameters.

Hence, our objective in this study is to design an acknowledgement scheme for S\&W ARQ that maximizes the latency-efficiency and/or energy-efficiency (i.e. minimizes the energy consumption) for data delivery in a multi-hop acoustic communication system. Quantitatively, for the delivery of a single data packet from node 1 to node $n+1$ for the system shown in Fig. 1(a), we evaluate the delivery time $\left(T_{d}\right)$, total time required for all transmissions to cease $\left(T_{c}\right)$ and the total number of bits transmitted $(B)$. The metric $T_{c}$ measures the latency-efficiency while $B$ measures the energy-efficiency of the acknowledgement scheme.

\section{OPPORTUNISTIC ACKNOWLEDGEMENT SCHEMES FOR MULTI-HOP ACOUSTIC COMMUNICATION}

To determine an optimally-efficient acknowledgement scheme, it is necessary to obtain analytic expressions for each metric in terms of the channel parameters. While it is straightforward to derive the packet delivery time $T_{d}$ analytically for a multi-hop communication system, it is complex to do so for $\left(T_{c}, B\right)$. Hence, we assume that the latter quantities may be approximately minimized by minimizing the corresponding per-hop quantities.

Let us assume that a new packet has just been received successfully at node $j+1$ from node $j$. With S\&W ARQ, the 


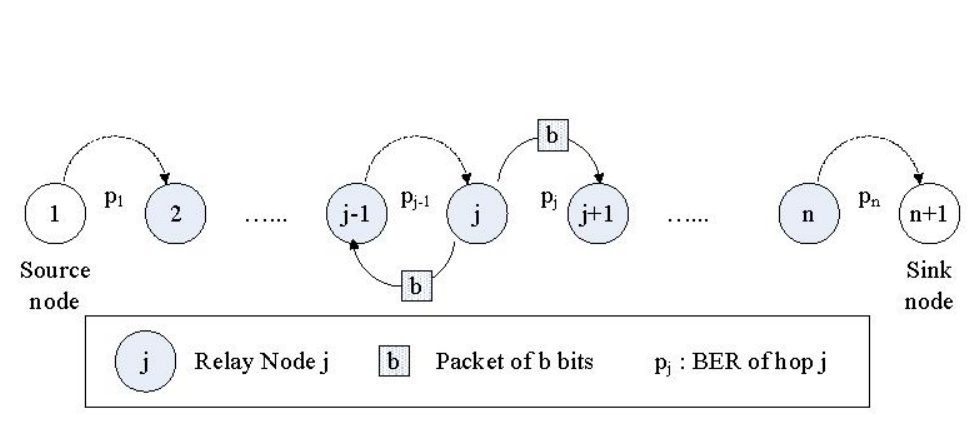

(a) One-dimensional $n$-hop acoustic channel

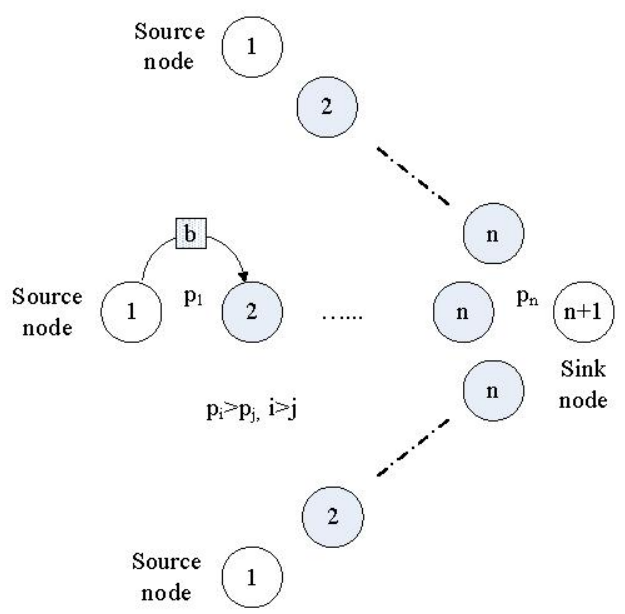

(b) Two-dimensional multi-hop acoustic sensor network

Fig. 1. (a) Illustration of Stop and Wait (S\&W) ARQ mechanism over a one-dimensional $n$-hop communication channel, where $p_{j}$ is the BER over the $j^{\text {th }}$ hop and (b) Extension to two-dimensional multi-hop network with single sink, where $p_{i}>p_{j}, i>j$.

above event has to be acknowledged at node $j$ before it can transmit the next packet. We quantify the per-hop latencyefficiency and energy-efficiency by the expected time elapsed and number of bits transmitted between node $j$ and $j+1$ until node $j$ has been acknowledged. We denote these quantities by $t_{j, \mathrm{~A}}$ and $b_{j, \mathrm{~A}}$ for acknowledgement scheme A respectively.

\section{A. Latency-efficient Opportunistic Acknowledgement}

Based on the mechanisms of IMP and EXP, we obtain the expressions for $t_{j, \mathrm{MPP}}$ and $t_{j, \mathrm{EXP}}$ as follows:

$$
\begin{aligned}
t_{j, \mathrm{IMP}} & =\frac{\tau_{O, \mathrm{IMP}}}{2} \frac{1+p_{j}^{2}\left(b_{A}\right)}{1-p_{j}^{2}\left(b_{A}\right)}+\left[t_{D}+t_{A}+2 t_{P}\right] p_{j}\left(b_{D}\right), \\
t_{j, \mathrm{EXP}} & =\frac{\tau_{O, \mathrm{EXP}}}{2} \frac{1+p_{j}^{2}\left(b_{A}\right)}{1-p_{j}^{2}\left(b_{A}\right)}-t_{D} p_{j}\left(b_{A}\right) .
\end{aligned}
$$

Hence, over hop $j$, we expect the IMP scheme to perform better in terms of latency-efficiency if the following condition holds:

$$
t_{j, \mathrm{IMP}}<t_{j, \mathrm{EXP}}
$$

If we assume that $p_{j}(b) \approx b \cdot p_{j}$ and $p_{j}^{2}\left(b_{A}\right)<<1$ (valid for $p_{j}<<1$, the above condition can be expressed in a simple form as follows:

$$
p_{j}<\frac{t_{A}}{\left(t_{D}+t_{A}+2 t_{P}\right) b_{D}+t_{D} b_{A}} \equiv p_{l}^{*} .
$$

Based on Eq. (1), we propose an opportunistic acknowledgement scheme, HYB-LAT, that exploits the spatial variance of acoustic channels to improve latency-efficiency using the following rule:

Rule 2: Whenever a relay node $j+1$ receives a packet from node $j$, it operates according to the following:

$$
\begin{aligned}
\text { If } \quad p_{j} \geq p_{l}^{*}, & \text { node } j+1 \text { performs EXP; } \\
\text { else } & \text { node } j+1 \text { performs IMP. }
\end{aligned}
$$

\section{B. Energy-efficient Opportunistic Acknowledgement}

As in Section IV-A, we can obtain the expressions for $b_{j, \mathrm{MMP}}$ and $b_{j, \mathrm{EXP}}$ as follows:

$$
\begin{aligned}
b_{j, \mathrm{IMP}} & =\frac{b_{D}+b_{D}\left[p_{j-1}\left(b_{D}\right)-p_{j}\left(b_{A}\right)\right]+b_{A} p_{j}\left(b_{D}\right)}{1-p_{j}\left(b_{A}\right)}, \\
b_{j, \mathrm{EXP}} & =\frac{b_{D}+b_{A}\left[1+p_{j}\left(b_{A}\right)-p_{j}^{2}\left(b_{A}\right)\right]}{1-p_{j}\left(b_{A}\right)} .
\end{aligned}
$$

Hence, we expect the IMP scheme to be more energy-efficient over hop $j$ if the following condition holds:

$$
b_{j, \mathrm{MMP}}<b_{j, \mathrm{EXP}}
$$

Using the same assumptions as in Section IV-A, the above condition can be expressed in terms of the system parameters as follows:

$$
p_{j}<\frac{b_{A}}{b_{D}^{2}-b_{A}^{2}} \equiv p_{e}^{*} .
$$

Using Eq. (2), we propose another opportunistic acknowledgement scheme, HYB-ENE, that improves energy efficiency using the following rule:

Rule 3: Whenever a relay node $j+1$ receives a packet from node $j$, it operates according to the following:

$$
\begin{aligned}
\text { If } \quad p_{j} \geq p_{e}^{*}, & \text { node } j+1 \text { performs EXP; } \\
\text { else } & \text { node } j+1 \text { performs IMP. }
\end{aligned}
$$

\section{Illustration of Opportunistic Acknowledgement Schemes}

To illustrate the operating region of our proposed opportunistic acknowledgement schemes over any hop, we consider a multi-hop acoustic communication channel with system parameters as given in Table I.

Using Eq. (1) and (2), we plot $\left(p_{l}^{*}, p_{e}^{*}\right)$ against $b_{D}$ in the LHS of Fig. 2. We observe that $p_{e}^{*}>p_{l}^{*}$, and we can 


\begin{tabular}{|l|c|}
\hline System parameters (bits) & Value \\
\hline$b_{D}$ (kbits) & $1-4$ \\
\hline$b_{A}$ (kbits) & $b_{D} / 20$ \\
\hline$c(\mathrm{~m} / \mathrm{s})$ & 1500 \\
\hline$l(\mathrm{~m})$ & 200 \\
\hline$r$ (kbps) & $5-7$ \\
\hline
\end{tabular}

TABLE I

Parameters to illustrate and evaluate the performance of Stop and Wait $A R Q$ protocols over the multi-hop acoustic systems in Fig. 1.

identify three operating regions in terms of $\left(b_{D}, p_{j}\right)$ described as follows:

\section{- RI: Apply IMP}

In this region, the opportunistic acknowledgement scheme selects IMP to achieve better performance in terms of both latency- and energy-efficiency.

\section{- RII: Apply EXP}

In this region, the opportunistic acknowledgement scheme selects EXP to achieve better performance in terms of both latency- and energy-efficiency.

- RIII: Trade-off between latency and energy-efficiency In this region, the preferred acknowledgement scheme depends on the relative importance of latency- and energy-efficiency. If the objective is to improve latencyefficiency, EXP should be applied; otherwise, IMP should be applied for better energy-efficiency.

\section{NUMERICAL RESULTS}

We compare the performance of our proposed opportunistic acknowledgement schemes against the traditional nonopportunistic IMP and EXP schemes for S\&W ARQ in terms of $\left(T_{d}, B, T_{c}\right)$ for the multi-hop acoustic communication systems in Fig. 1 using the parameters defined in Table I.

\section{A. Spatially-invariant channel}

We consider a one-dimensional $n$-hop acoustic channel that is suitable for long-range sensing / surveillance applications, as shown in Fig. 1(a). We assume that the channel is spatiallyinvariant (i.e., $\mathrm{BER} p_{j}=p$ ), simulate the delivery of a single data packet $\left(b_{D} \mathrm{Kbits}\right)$ and evaluate $\left(T_{d}, B, T_{c}\right)$ averaged over 10000 runs.

To demonstrate the effectiveness of our proposed opportunistic acknowledgement schemes, we consider $5 \times 10^{-6} \leq p \leq 5 \times 10^{-5}$ which is within the typical interval $\left[10^{-6}, 10^{-2}\right]$ [11] for acoustic channels and also includes $\left(p_{l}^{*}, p_{e}^{*}\right)$ as given in the LHS of Fig. 2 .

The results obtained for $n=6$ and $b_{D}=2$ Kbits are shown in the RHS of Fig. 2 and Fig. 3, alongside the corresponding values of $\left(p_{l}^{*}, p_{e}^{*}\right)$ used by the HYB-LAT and HYB-ENE schemes respectively.

As expected, the performance of each scheme is degraded as the channel quality is worsened. For $B$ and $T_{c}$, we observe that there exists a crossover point (denoted by $p^{*}$ ) for which
IMP outperforms EXP when channel conditions are better ( $p$ $<p^{*}$ ) and vice versa. Since HYB-LAT and HYB-ENE select IMP when channel conditions are better and EXP otherwise, optimal performance can be achieved with HYB-LAT (HYBENE) if $p_{l}^{*}=p^{*}\left(p_{e}^{*}=p^{*}\right)$. In fact, we observe that $p_{l}^{*} \approx p^{*}$ and therefore, HYB-LAT gives the best performance in terms of $\left(B, T_{c}\right)$ for the range of channel conditions considered.

On the other hand, such a crossover point does not exists for the metric $T_{d}$, and IMP delivers the packet within a shorter duration than EXP under all channel conditions. This is expected since $T_{d}$ depends only on the per-hop transmission and propagation delay and is independent of the acknowledgement scheme employed. Since an ACK packet is piggy-backed to each data packet with EXP, it incurs a larger transmission delay $\left(t_{D}+t_{A}\right)$ compared with $\mathrm{IMP}\left(t_{D}\right)$ and therefore, each packet will be delivered quicker with IMP. Hence, while HYB-LAT also gives the best performance in terms of $T_{d}$ when channel conditions are good, there is a trade-off between the metrics $T_{d}$ and $\left(B, T_{c}\right)$ when channel conditions worsen.

\section{B. Spatially-variant channel}

Next, we consider a more realistic spatially-variant (i.e., $p_{i} \neq p_{j}$ ) one-dimensional $n$-hop acoustic channel, where $p_{j}$ is uniformly distributed in the interval $\left[p-0.4 \times 10^{5}, p+0.4 \times 10^{5}\right]$, where $p$ is the mean BER over the $n$-hops. The corresponding results of Fig. 3 for the spatially-invariant random channel are plotted in Fig. 4.

Finally, we extend the one-dimensional string topology in Fig. 1(a) to two dimensions, where several one-dimensional multi-hop communication channels converge to a common sink to form a two-dimensional network, as shown in Fig. 1(b). With such a topology, the channel quality is likely to degrade towards the sink due to convergence of data delivery near the sink. Accordingly, we define a suitable spatially-variant linear channel model with mean BER over the channel of $p$ as follows:

$$
p_{j}=p+0.2 \times 10^{-5} \cdot\left(j-\frac{n}{2}\right) .
$$

The corresponding results for the spatially-variant linear channel are plotted in Fig. 5. Similar trends are observed between the spatially-invariant and spatially-variant channels (both random and linear), with the HYB-LAT acknowledgement scheme giving the best performance amongst all the schemes considered.

\section{CONCLUSIONS AND FUTURE WORK}

Underwater acoustic networks are envisaged to be the enabling technology for oceanographic data collection, pollution monitoring, offshore exploration and tactical surveillance applications. Although reliable and efficient communication protocols are in place for terrestrial networks, they cannot be directly employed in underwater environment due to the unique characteristics of underwater acoustic channels such as higher bit error rates and larger propagation delays. As a result, packet losses are significant, and the design of efficient ARQ 

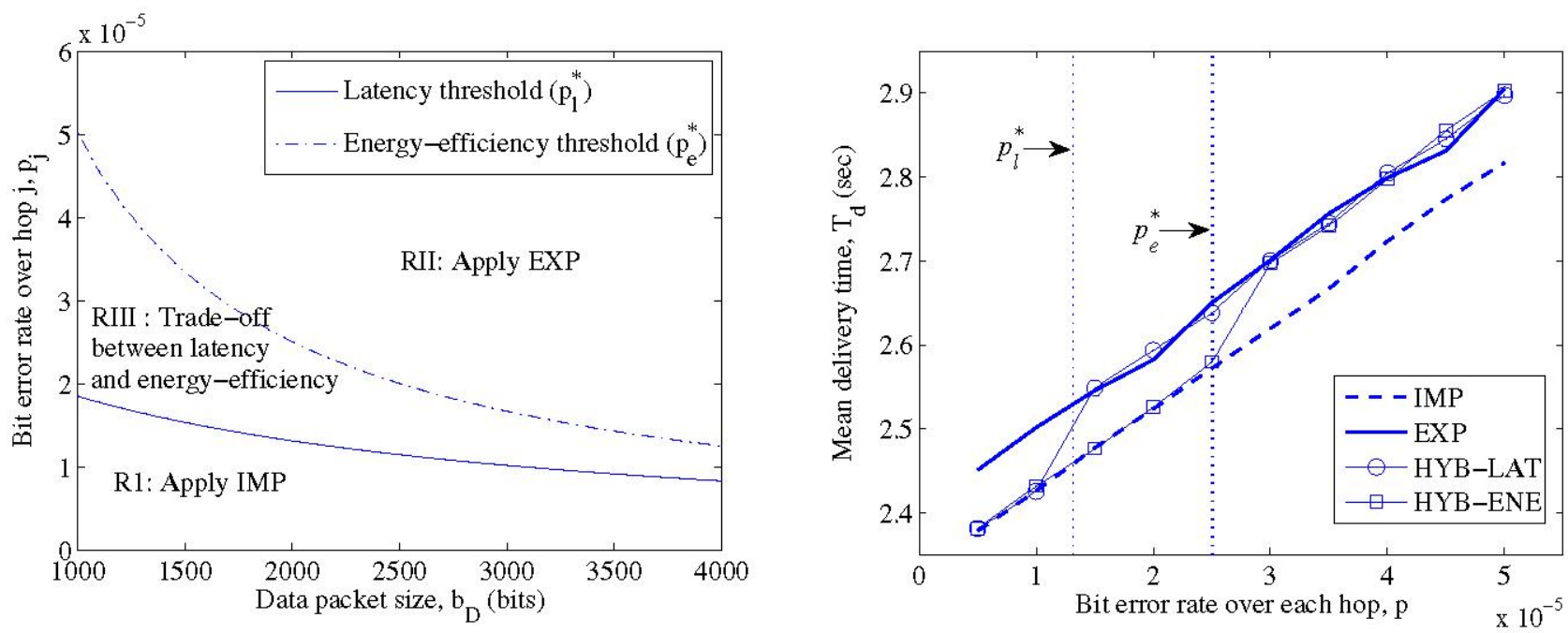

Fig. 2. Operating region $\left(b_{D}, p_{j}\right)$ of opportunistic acknowledgement schemes HYB-LAT and HYB-ENE (left) obtained for the system parameters in Table I; Mean packet delivery time, $T_{d}$ under a spatially-invariant channel for $n=6$ and $b_{D}=2 \mathrm{Kbits}$ (right).
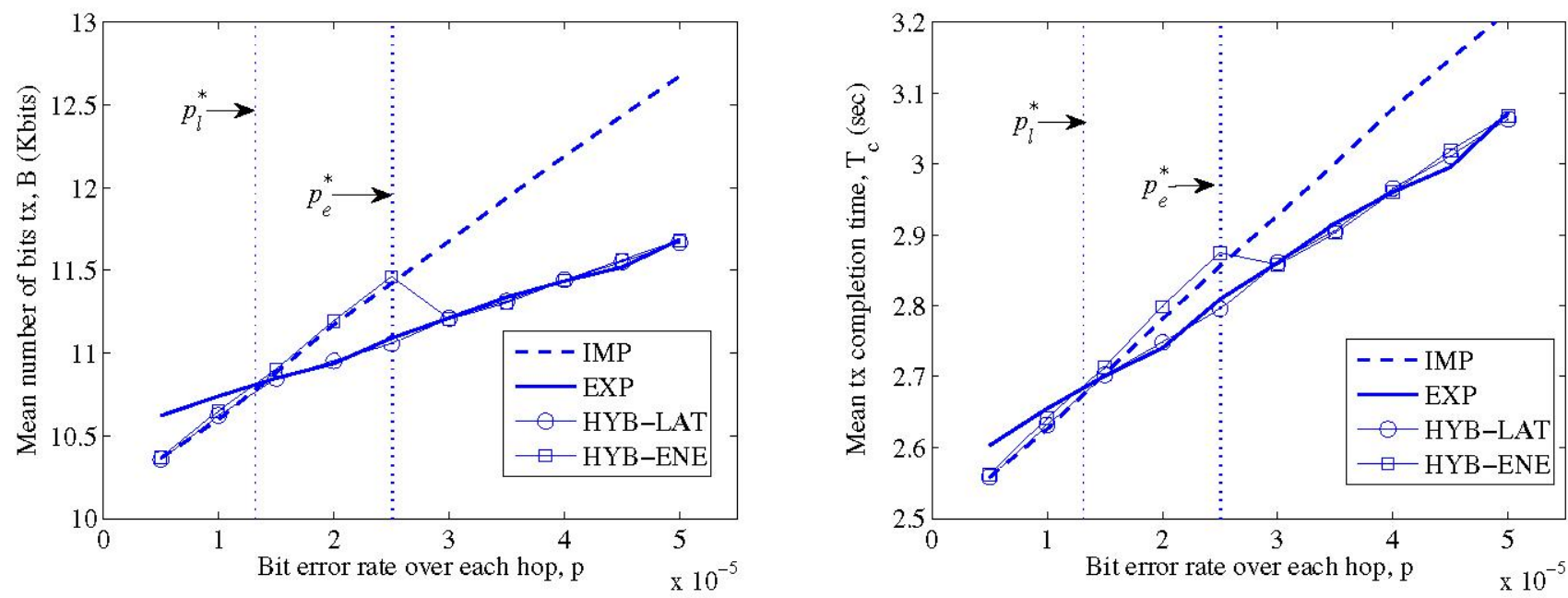

Fig. 3. Mean number of bits transmitted, $B$ (left) and transmission completion time, $T_{c}$ (right) for a spatially-invariant channel for $n=6$ and $b_{D}=$ 2 Kbits.

schemes to ensure reliability through packet retransmissions and acknowledgements is important.

In this paper, we proposed an opportunistic acknowledgement scheme suited for Stop and Wait ARQ protocols and demonstrated using simulations that it can offer better latency and energy efficiency than traditional non-opportunistic schemes for both one and two-dimensional multi-hop acoustic channels.

While we have considered single packet transmissions in this study, the opportunistic acknowledgement scheme may be applied for transmission of a group of packets [9] to achieve better performance. In addition, we also plan to incorporate this scheme for the comparison of a single path routing scheme with ARQ and a multi-path redundancy routing scheme for the virtual sink architecture proposed in [12].

\section{REFERENCES}

[1] I. F. Akyildiz, D. Pompili, and T. Melodia, "Underwater Acoustic Sensor Networks: Research Challenges," Elsevier Journal of Ad Hoc Networks, vol. 3, no. 3, pp. 257-279, March 2005.

[2] J. Heidemann, W. Ye, J. Wills, A. Syed, and Y. Li, "Research challenges and applications for underwater sensor networking," Proc. of IEEE WCNC, pp. 228-235, April 2006.

[3] A. Sastry, "Improving automatic repeat request (ARQ) performance on satellite channels under high error rate conditions," IEEE Trans. Commun., vol. 23, pp. 436-439, April 1975.

[4] M. Moeneclaey, H. Bruneel, I. bruyland, and D. Chung, "Throughput optimization for a generalized Stop-and-Wait ARQ scheme," IEEE Trans. Commun., vol. 34, pp. 205-207, February 1986.

[5] J. M. Morris, "Optimal blocklengths for ARQ error control schemes," IEEE Trans. Commun., vol. 27, pp. 488-493, February 1979.

[6] P. F. Turney, "An improved Stop-and-Wait ARQ logic for data transmission in mobile radio systems," IEEE Trans. Commun., vol. 29, pp. 68-71, January 1981. 

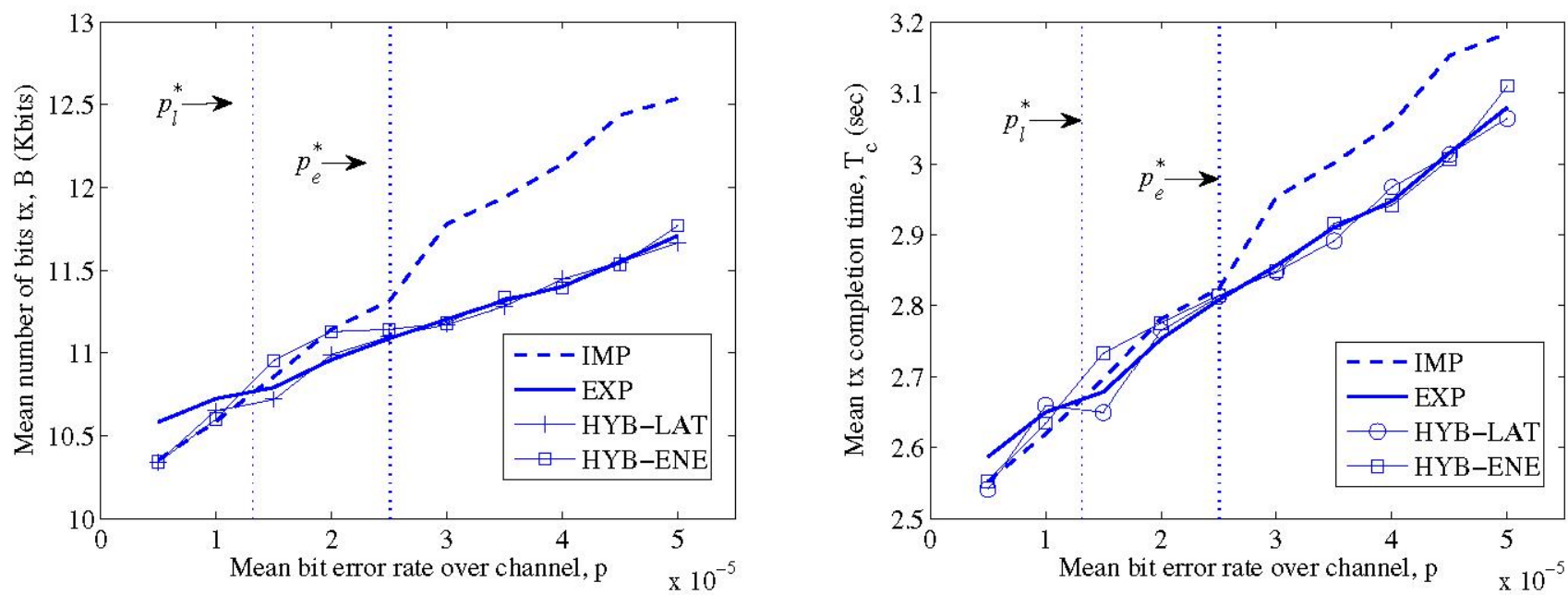

Fig. 4. Mean number of bits transmitted, $B$ (left) and transmission completion time, $T_{c}$ (right) for a spatially-variant random channel (mean BER $=$ p) for $n=6$ and $b_{D}=2 \mathrm{Kbits}$.
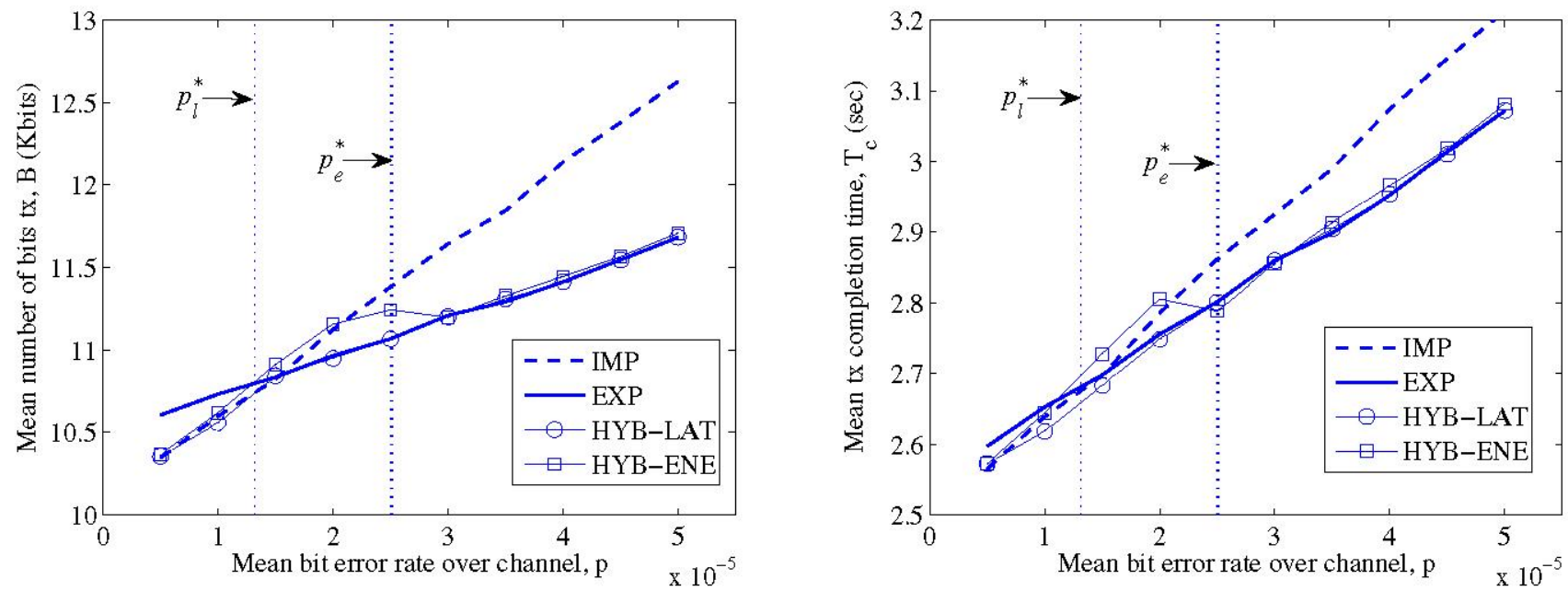

Fig. 5. Mean number of bits transmitted, $B$ (left) and transmission completion time, $T_{c}$ (right) for a spatially-variant channel (mean $B E R=p$ ) for $n=6$ and $b_{D}=2 \mathrm{Kbits}$.

[7] H. Wiemann, M. Meyer, R. Ludwig, and P. O. Chang, "A Novel MultiHop ARQ Concept," Proceedings of the IEEE VTC, vol. 5, pp. $3097-$ 3101, May 2005.

[8] M. Lott, "ARQ for Multi-Hop Networks," Proc. of the IEEE VTC, vol. 3, pp. 1708-1712, September 2005.

[9] M. Stojanovic, "Optimization of a Data Link Protocol for an Underwater Acoustic Channel," Proc. of the IEEE Oceans Europe, vol. 1, pp. 68-73, June 2005.

[10] D. Pompili, T. Melodia, and I. F. Akyildiz, "Routing algorithms for delay-insensitive and delay-sensitive applications in underwater sensor networks," Proc. of the ACM MOBICOM, pp. 298-309, September 2006.

[11] E. Sozer, M. Stojanovic, and J. Proakis, "Underwater Acoustic Networks," IEEE Journal of Oceanic Engineering, vol. 25, no. 1, pp. 72-83, January 2000.

[12] W. K. G. Seah and H. P. Tan, "Multipath virtual sink architecture for wireless sensor networks in harsh environments," Proc. of the ACM Intersense, vol. 138, May 2006, invited paper. 